\title{
Fluorescein iridography
}

\author{
R. MAPSTONE
}

Department of Ophthalmology, University of Liverpool, and St. Paul's Eye Hospital, Liverpool

The behaviour of anterior segment inflammation, as regards its temporal aspects, can be $\vec{\circ}$ objectively assessed thermometrically (Mapstone, 1968); conclusions of spatial distribution can, only be inferred. If the extent of inflammation could be demonstrated, a correlation of temporal pattern with spatial distribution would allow a more precise $\frac{0}{0}$. interpretation of the clinical characteristics of anterior uveitis with attendant diagnostic, or prognostic, and therapeutic gain. The purpose of this paper is to describe a method of investigating the spatial distribution of anterior uveitis, and the intensity of its vascular component, based on the reaction of normal and pathological iris vessels to injected fluorescein.

\section{Material and methods}

A Zeiss fundus camera and flash unit with an Ilford bright spectrum blue No. 622 gelatine filter for excitation and a Kodak Wratten 15 yellow filter as barrier was used for angiography. Photographs were taken prior to intravenous injection of $3 \mathrm{ml}$. 25 per cent. fluorescein, and Io sec. later, at $2 \mathrm{sec}$. intervals for $40 \mathrm{sec}$. Final photographs were taken $5 \mathrm{~min}$. after injection.

The material consisted of 48 normal subjects, 44 patients with vein occlusions, 68 patients with anterior uveitis, and seventeen with miscellaneous ocular conditions.

\section{Results}

NORMAL ANGIOGRAM

\section{(A) Preinjection fluorescence}

Control photographs taken before injection show either no image or a vague outline of lids and periocular tissue. The lens, however, shows a variable fluorescence which is most marked in the elderly (Fig. I, opposite).

\section{(B) Postinjection fluorescence}

The sequence of photographs in Fig. 2 shows the anterior segment response to injected $\frac{D}{2}$ fluorescein, viz. pupillary fluorescence due to retinal vessel filling followed by a gradual leak into conjunctiva and skin. Pupillary fluorescence is dependent upon a relatively dilated pupil and lessens or disappears after the first dye transit. The normal iris exhibits one of the following patterns:

(r) No fluorescence, more common in younger patients whose stromal tissue has not yet atrophied. It is not dependent on degree of pigmentation since even blue irides may not fluoresce.

(2) Transient fluorescence during the first dye transit (Fig. 3, overleaf).

(3) Vessel fluorescence during the first dye transit, which then regresses and is followed by a perceptible blush of vessels and iris stroma (Fig. 4, overleaf). 
FIG. I Fluorescence of ageing lens, pre-injection photograph

(a)
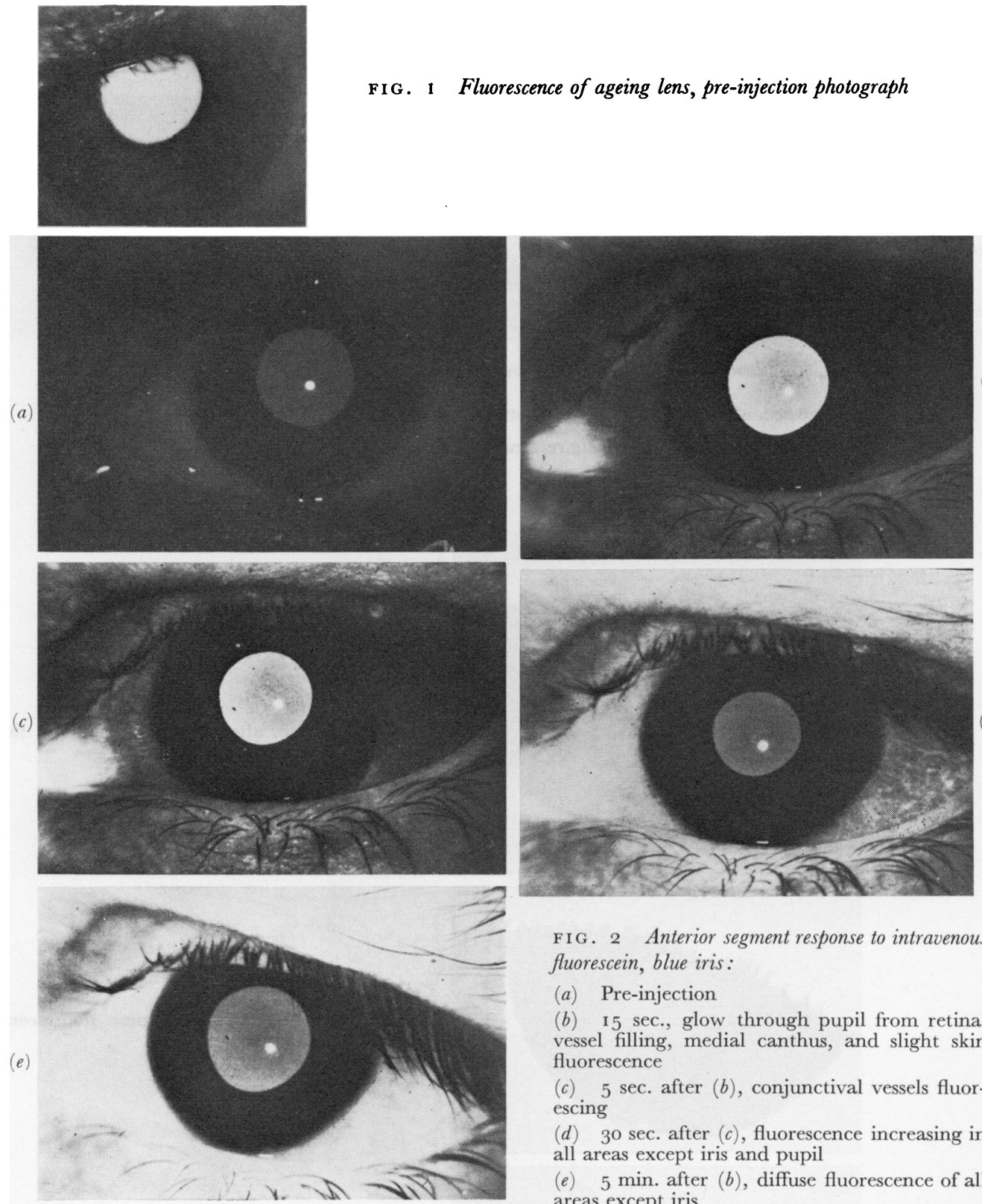

FIG. 2 Anterior segment response to intravenous fluorescein, blue iris:

(a) Pre-injection

(b) I 5 sec., glow through pupil from retinal vessel filling, medial canthus, and slight skin fluorescence

(c) 5 sec. after $(b)$, conjunctival vessels fluorescing

(d) 30 sec. after (c), fluorescence increasing in all areas except iris and pupil

(e) $5 \mathrm{~min}$. after $(b)$, diffuse fluorescence of all areas except iris

Normal iris fluorescence shows the following characteristics:

(a) The first part of the anterior segment to fluoresce is the caruncle; a time lag of up to $8 \mathrm{sec}$. elapses before iris vessels fluoresce.

(b) The number of vessels fluorescing increases with time.

(c) Not all vessels (or what appear to be vessels) fluoresce.

(d) Leakage into the iris stroma (as distinct from vessel wall) is minimal, and does not occur during the first dye transit.

(e) Little aqueous fluorescence is seen in photographs. 


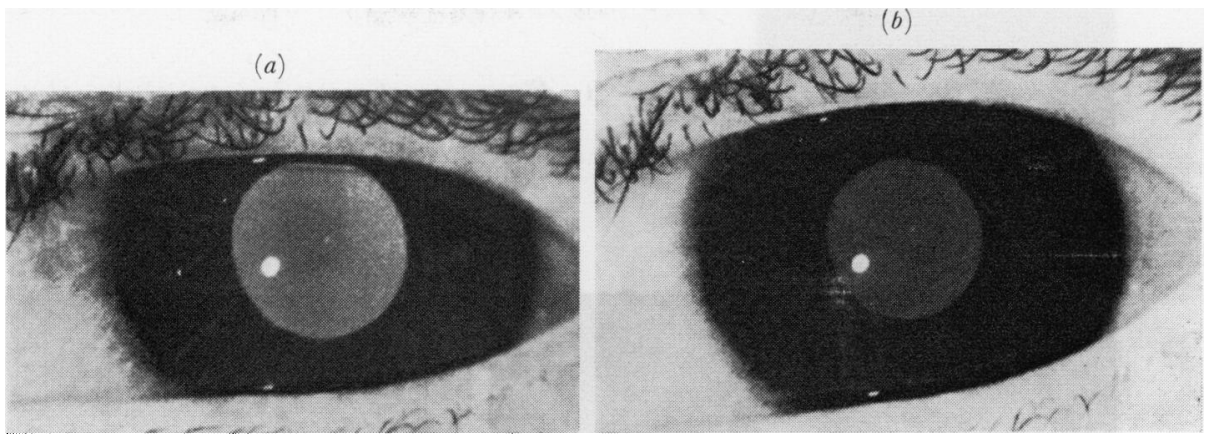

FI G. 3 Fluorescence during first dye transit, blue iris:

(a) $20 \mathrm{sec}$. after injection, vague fluorescence of iris vessels

(b) 40 sec. after (a), no iris fluorescence

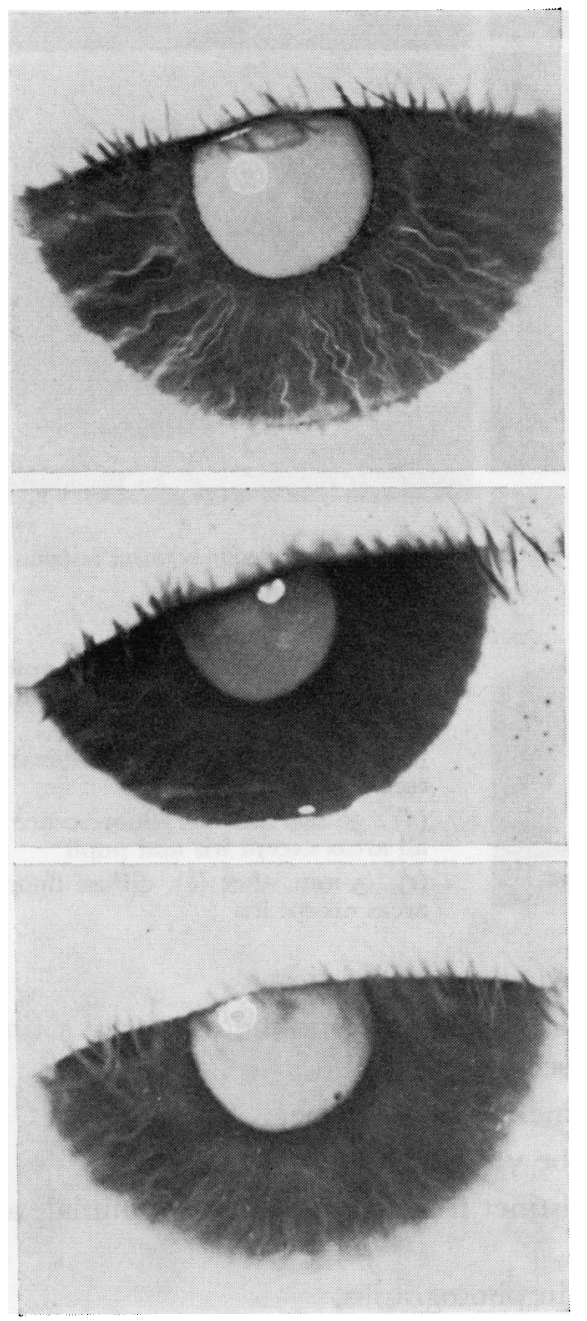

(a) 20 sec. after injection, maximal fluorescence of iris vessels

(b) 6o sec. after (a), some fluorescence of iris vessel wall

(c) 5 min. after (a), light, diffuse fluorescence of vessels and stroma, excluding area between pupil margin and collarette, i.e. true tissue fluorescence not due to aqueous haze

FIG. 4 Biphasic response of normal blue iris 
ABNORMAL ANGIOGRAM

\section{(A) Lightly pigmented irides}

Inflamed and new vessels both fluoresce; Fig. 5 shows a sequence from a patient with neovascularization secondary to proliferative diabetic retinopathy.

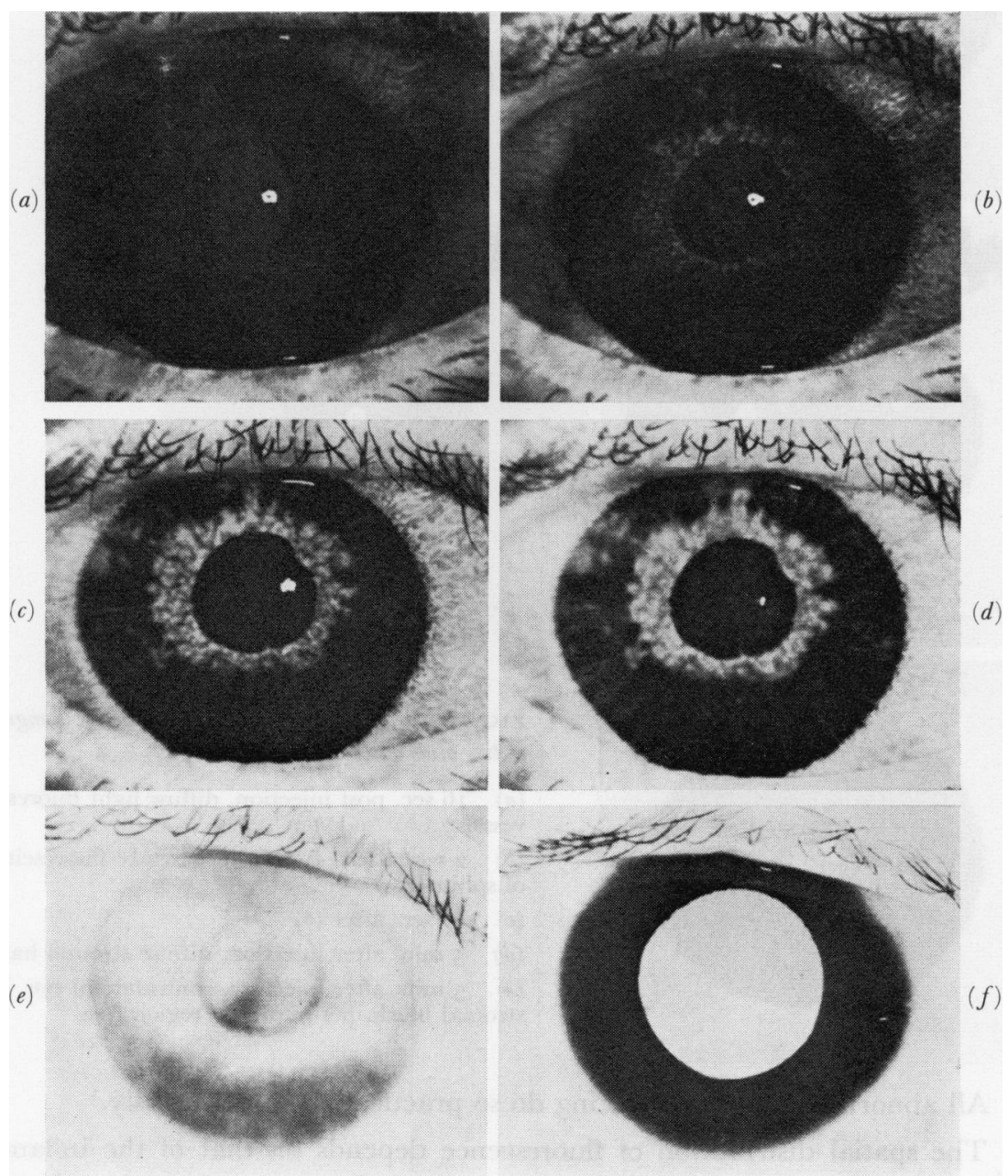

FIG. 5 Fluorescence secondary to neovascularization (proliferative diabetic retinopathy)

(a) $17 \mathrm{sec}$. after injection, light fluorescence of conjunctiva and periorbital skin

(b) 2 sec. after $(a)$, new vessels leaking into stroma before normal iris vessels fluoresce

(c) 6 sec. after $(b)$

(d) $8 \mathrm{sec}$. after $(b)$ diffuse peripupillary blush with blush of normal iris vessel walls

(e) 5 min. after injection

(f) $5 \mathrm{~min}$. after injection, contralateral eye, no iris blush. Note intense pupillary fluorescence from retinal new vessels

Fig. 6 (overleaf) shows a sequence from a patient with diffuse iridocyclitis. It has the following characteristics:

(a) A variable time lag between conjunctival and iris fluorescence. 


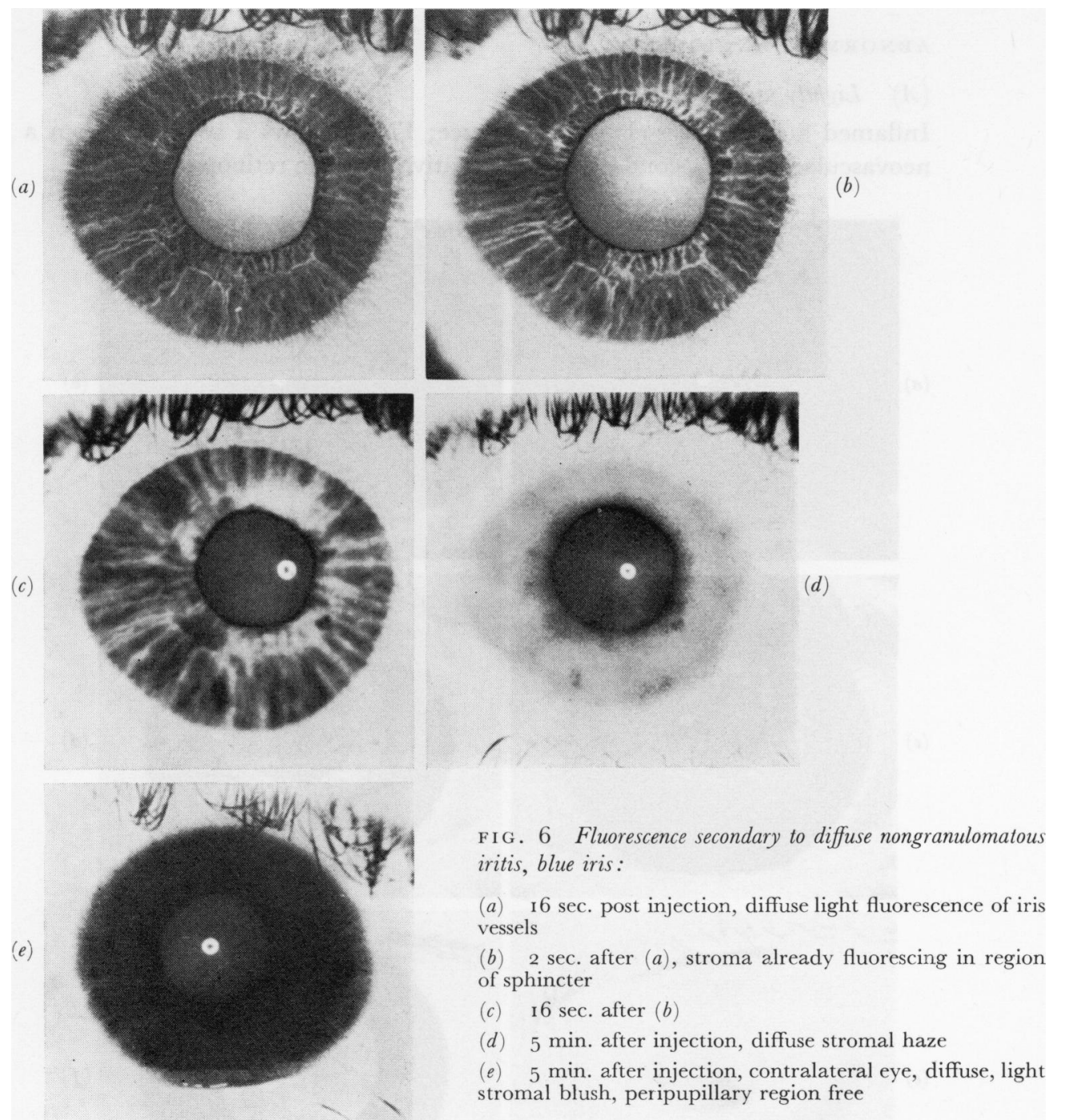

(b) All abnormal vessels fluorescing do so practically simultaneously.

(c) The spatial distribution of fluorescence depends on that of the inflammation, but even in a diffuse reaction not all (putative) vessels fluoresce. In early rubeosis the spatial distribution varies with the extent of neovascularization; in late rubeosis both normal and new vessels leak.

(d) Fluorescence increases with time.

(e) Diffusion into surrounding stroma occurs during the first dye transit, followed by diffuse aqueous fluorescence.

(B) Heavily pigmented irides

(I) Negroid and mongoloid

Little of value can be obtained from these angiograms, there being either a fluorescein leak from the pupil margin or a diffuse aqueous haze. 


\section{(2) Caucasoid}

Pigmentation is usually not of uniform density and through its interstices fluorescence can be seen. From the distribution and time sequence conclusions can be drawn based on the more obvious behaviour of lightly pigmented irides (Fig. 7).

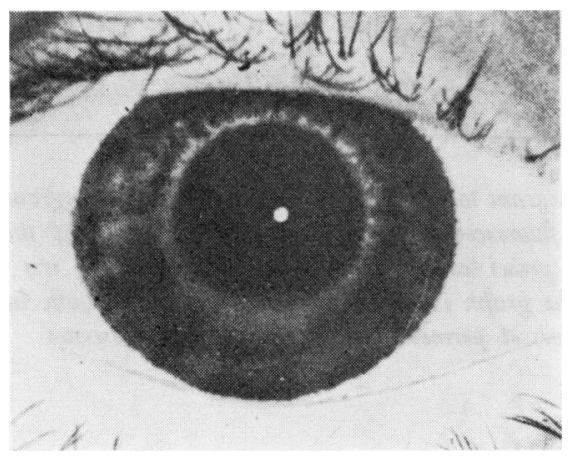

FIG. 7 Fluorescence secondary to diffuse nongranulomatous iridocyclitis, Caucasian, brown iris. Photograph 24 sec. after injection, i.e. fluorescence can only have come from inflamed vessels

\section{Discussion}

Normal anterior uvea leaks fluorescein in sufficient quantities to be measured by a fluorometer (Amsler and Huber, I946; Langham and Wybar, 1954). Photographically two distinct types of fluorescence have to be distinguished, viz. intravascular due to the first dye transit and extravascular dependent upon a leak of fluorescein into the vessel wall and stroma.

The photographic detection of intravascular fluorescence is determined-amongst other things-by:

(I) A patent vessel

(2) Thickness and pigmentation of vessel wall

(3) Pigmentation of anterior border layer of iris

(4) Concentration of fluorescein

The temporal characteristics of this type of fluorescence are illustrated in Fig. 8, i.e. fluorescence during the first dye transit only (see Fig. 3).

Any further fluorescence in an iris angiogram must be extravascular, within the vessel wall, iris stroma, or aqueous. Its photographic detection will depend on the abovementioned factors and in addition upon vessel wall and endothelial permeability, the latter being the limiting factor. Its temporal characteristics are illustrated in Fig. 9, the gradient of the graph depending largely on endothelial permeability.

There may, however, be a combination of both intra- and extravascular fluorescence and if endothelial permeability is increased, as in an inflamed vessel, the intravascular component will be "swamped" by a rapid fluorescein leak (Fig. 5) and the pattern of Fig. 9 will be produced. Again, in some patients, if endothelial permeability is not pathologically increased, the pattern of fluorescence shown in Fig. 10 is produced, i.e. fluorescence during the first dye transit followed by a gradual extravascular fluorescence (Fig. 4).

The essential difference, therefore, between a normal and abnormal iris vessel is the leak of fluorescein from the latter during the first dye transit, allowing a ready demonstration of the spatial distribution of anterior uveal pathology. 


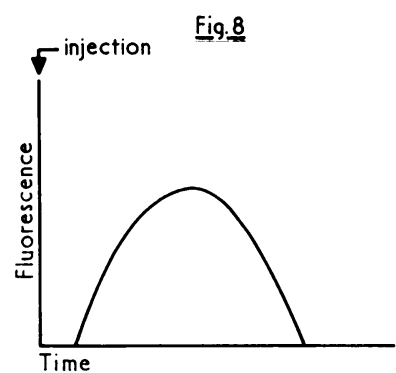

FIG. 8 Diagram to illustrate intravascular fluorescence of normal iris vessels during first dye transit

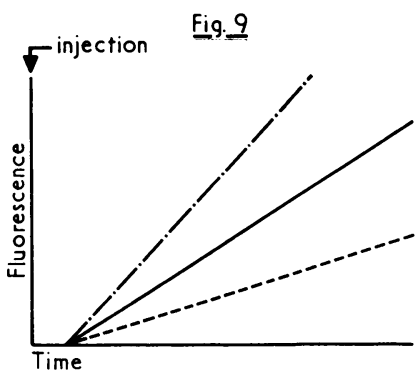

FIG. 9 Diagram to illustrate extravascular fluorescence of iris stroma due to vessel leak. The gradient of the graph is dependent on iris vessel permeability

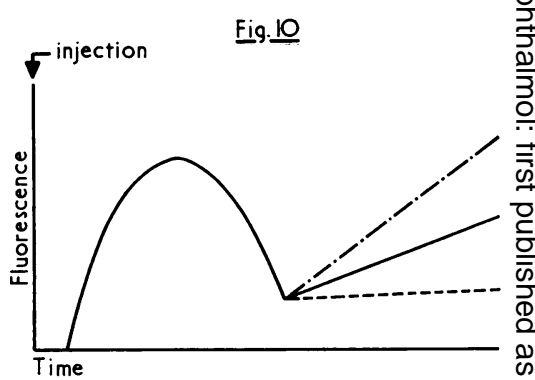

FIG. 10 Diagram to illustrate $\vec{\circ}$ biphasic pattern of fluorescence shown by some normal iris vessels, i.e. a combination of both intra- and extra- 응 vascular fuorescence

\section{Summary}

A method of demonstrating the spatial distribution of anterior uveal pathology based on $ᄋ$ the reaction of normal and abnormal iris vessels to injected fluorescein is described and discussed.

I should like to thank the surgeons of St. Paul's Eye Hospital for access to their patients, and Mr. R. McBride $\vec{\varphi}$ for technical help.

\section{References}

AMSLER, M., and HUBER, A. (1946) Ophthalmologica (Basel), III, I 55

LANGHAm, м., and WYBAR, к. c. (1954) Brit. 7. Ophthal., 38, 52

MAPSTONE, R. (1968) Ibid., 52, 9 I 7 